\title{
A POLÍTICA DE EDUCAÇÃO PARA A SAÚDE EM PORTUGAL
}

\author{
Denise Capela dos Santos
}

ORCID ID:0000-0002-5794-5203;

CIÊNCIA ID: 0B10-1C58-71EB,

dsantos@autonoma.pt

Em Março deste ano, o Governo em atuais funções criou o Programa Nacional de Educação para a Saúde, Literacia e Autocuidados (PNESLA), associado à:

1. preparação e apoio dos prestadores informais em cuidados domiciliários,

2. prevenção da diabetes,

3. prevenção da obesidade,

4. promoção da saúde mental,

5. promoção do envelhecimento saudável, e da

6. utilização racional e segura do medicamento.

A implementação deste programa no território português, prevista até Junho de 2017, deverá ter um papel determinante no envolvimento dos cidadãos no sistema de saúde, na co-responsabilização dos cidadãos pelo seu estado de saúde, na adopção de estilos de vida saudáveis, na prática correta de auto-cuidados e no ensino de familiares sem formação em saúde que tratam de doentes no domicílio. Esta implementação fica a cargo do Diretor Geral da Saúde, a nível nacional, dos Departamentos de Saúde Pública das Administrações Regionais de Saúde, a nível regional, e das Unidades de Saúde Pública, a nível dos Agrupamentos dos Centros de Saúde (ACES) / Unidades Locais e Saúde (ULS), a nível local. Estão previstos controlos semestrais do processo (Augusto e Fronteira, 2016).

Esta "nova ambição para a Saúde Pública" baseia-se na premissa de que a educação, a literacia e os auto-cuidados são essenciais para a promoção da saúde, para a proteção da doença e para a eficácia e eficiência dos cuidados de saúde (Augusto e Fronteira, 2016).

Estes objetivos estão enquadrados nas metas para 2020 estabelecidas no Plano Nacional de Saúde 2012-2016-2020, de: redução da taxa de mortalidade prematura (\%) antes dos 70 anos de idade, aumento da esperança de vida saudável aos 65 anos de idade, redução da prevalência do consumo de tabaco na população com $\geq 15$ anos e eliminação da exposição ao fumo ambiental, e controlo da incidência e da prevalência de excesso de peso e obesidade na população infantil e escolar (DGS, 2015)a.

Em Maio, o atual Governo definiu como programas de saúde prioritários, no Despacho n.. 6401/2016 de 16 de Maio, dois programas relacionados com a promoção da saúde: o Programa de Promoção da Alimentação Saudável e o de Promoção da Atividade Física. No mesmo 
despacho, fixou dois programas de prevenção da doença: o Programa de Prevenção e Controlo do Tabagismo e o Prevenção e o Programa de Controlo de Infeções e de Resistência aos Antimicrobianos. Atribuiu ainda importância aos Programas Nacionais da Diabetes e de Saúde Mental, que incluem estratégias de promoção da saúde e de prevenção das respetivas doenças (DGS, 2016)a . A determinação destes Programas, enquadrados no âmbito do Plano Nacional de Saúde, atribui especial relevância à necessidade de promover a saúde e de prevenir a doença, antes da necessidade de proceder ao seu tratamento, evitando tempo de vida com incapacidade associado à doença e custos desnecessários para as famílias e para o Estado. Esta função deverá estar principalmente a cargo dos profissionais de saúde das Unidades de Saúde Familiares e das Unidades de Cuidados de Saúde Personalizados, dos ACES /ULS, das farmácias comunitárias e das autarquias (em parceria com os anteriores).

Considera-se que o programa prioritário de Promoção da Alimentação Saudável, vai estar associado ao PNESLA relativamente à prevenção da diabetes e da obesidade, e à promoção do envelhecimento saudável. O Programa de Promoção da Atividade Física irá estar associado à prevenção da diabetes e da obesidade, à promoção do envelhecimento saudável e à promoção da saúde mental. Já o Programa de Prevenção e Controlo do Tabagismo é importante para a promoção do envelhecimento saudável, enquanto os Programas Nacionais da Diabetes e da Saúde Mental terão impacto na promoção da saúde da população e na prevenção, tratamento e reabilitação da diabetes e das doenças mentais, respetivamente (SNS, 2016) $)^{\text {a }}$ Por fim, o Programa de Controlo de Infeções e de Resistência aos Antimicrobianos terá impacto no uso racional de medicamentos de apenas um grupo terapêutico específico, do qual fazem parte os antibióticos, antivirais, antifúngicos e antiparasitários.

Nenhum destes programas se associa, no entanto, à preparação e apoio dos prestadores informais em cuidados domiciliários, nem à utilização segura e racional dos medicamentos na generalidade. Também não há nenhum específico para a promoção do envelhecimento saudável.

Vejamos os dados encontrados sobre ações que se colocaram em prática, no último ano, para prossecução dos objetivos do PNESLA.

\section{APOIO DE PRESTADORES INFORMAIS EM CUIDADOS DOMICILIÁRIOS}

As famílias assumem um papel essencial no apoio aos seus membros com doenças crónicas altamente incapacitantes e aguardam pela implementação de medidas reabilitativas, às quais a Rede Nacional de Cuidados Continuados Integrados nem sempre consegue dar resposta. A Direção-Geral da Saúde, através do Programa Nacional para a Saúde Mental, e a "Familiarmente", Federação Nacional das Associações das Famílias de Pessoas com Experiência em Doença Mental, organizaram o $1^{\circ}$ Encontro Internacional sobre o Papel e a 
Importância da Família na Doença Mental, que decorreu em Maio deste ano, em Lisboa (DGS, 2016) ${ }^{k}$, revelando preocupação com o apoio dos prestadores informais.

Um outro exemplo a salientar relaciona-se com a visita de uma equipa de técnicos da Unidade de Saúde Pública do ACES Lisboa Norte à Instituição Particular de Solidariedade Social Lar de Idosos Inválidos do Comércio, associada a um projeto, no âmbito do Plano de Inverno, de intervenções pedagógicas a estruturas residenciais coletivas para sensibilizar os colaboradores destas unidades para a necessidade de preparação adequada para o frio e para a gripe (DGS, 2016) $)^{\mathrm{s}}$.

\section{PREVENÇÃO DA DIABETES}

O Programa Nacional para a Promoção da Alimentação Saudável apresenta um contributo inicial para uma discussão alargada sobre a necessidade, benefícios e estratégias disponíveis para a redução do consumo de açúcar em Portugal, pretendida pelo Governo (DGS, 2016) c. Neste domínio, a "Direcção-Geral da Saúde entregou ao Ministério da Saúde uma proposta para reduzir para metade ou menos de metade (dos actuais oito gramas para apenas quatro ou três gramas) a quantidade de açúcar das embalagens individuais servidas na restauração" e sugere, ainda, que seja obrigatório que estes pacotes apenas sejam disponibilizados aos clientes que o pedirem (DGS, 2016) . Esta proposta segue as recomendações da Organização Mundial da Saúde (OMS), que defende que o consumo de açúcar se deve manter abaixo de 10\% (idealmente 5\%) das calorias ingeridas diariamente. De acordo com esta fonte, o consumo de açúcar pelas crianças portuguesas aproxima-se dos $25 \%$ da energia ingerida diariamente (DGS, 2016) ${ }^{\mathrm{r}}$, sugerindo que é urgente intervir. Como será do conhecimento público, a redução acentuada do consumo de açúcar é essencial à prevenção da diabetes, doença que se caracteriza por níveis de glicemia elevados.

O diretor do Programa Nacional para a Promoção da Atividade Física (PNPAF), da Direção-Geral da Saúde, explicou porque é que a atividade física é uma verdadeira prioridade de Saúde Pública na comunicação social $(D G S, 2016)^{d}$. Esta divulgação é importante para a prevenção e tratamento da diabetes, uma vez que a atividade física é determinante para ajudar à metabolização do açúcar no organismo e para a consequente redução do teor de glicose no sangue.

Por outro lado, a Associação Protectora dos Diabéticos de Portugal (APDP) tem um papel importante na promoção da saúde e prevenção da diabetes. Para tal, desenvolve anualmente vários cursos gratuitos de formação dirigidos a pessoas com diabetes, familiares e cuidadores, sobre as mais variadas temáticas relacionadas com a diabetes (DGS, 2016)p , promovendo também assim apoio a cuidadores informais, responsáveis pela confeção de alimentos de familiares diabéticos.

Outro projeto interessante neste campo foi o desenvolvimento de uma plataforma eletrónica para complemento da prescrição de exercício físico para doentes com diabetes tipo 2, pela Universidade de Trás-os-Montes e Alto Douro. Esta plataforma foi desenvolvida no contexto do 
Working Paper

Departamento de Ciências Económicas e Empresariais

Universidade Autónoma de Lisboa

projeto "Diabetes em Movimento", lançado em 2014, que visa promover o exercício físico e combater o sedentarismo nos doentes com diabetes (DGS, 2016) .

\section{PREVENÇÃO DA OBESIDADE}

A referência e reflexão sobre o PNPAF, pela Direção-Geral da Saúde, num jornal com notoriedade em Portugal foi importante para alertar a população com obesidade para a importância da prática de atividade física (DGS, 2016) ${ }^{d}$ que deve, nestes doentes, ser alvo de acompanhamento profissional. De facto, para que haja redução de peso, o teor de calorias dos alimentos ingeridos tem de ser inferior à energia gasta pelo indivíduo em atividade física e fisiológica. É por isso fundamental, para controlar e reduzir o índice de obesidade, apostar simultaneamente na atividade física e numa alimentação racional e saudável, considerando as calorias que cada um necessita de ingerir diariamente.

Neste sentido, com o apoio da DGS, avaliaram-se, em 2016, 29 programas de promoção de alimentação saudável e/ou atividade física realizados entre 2001 e 2015 em Portugal, permitindo reunir um conjunto de informação sobre a realidade nacional na área da prevenção e controlo da obesidade infantil $(D G S, 2016)^{e}$. Esta ação possibilita a caracterização das necessidades no território nacional relativamente a esta patologia e uma melhoria da eficiência dos projetos escolhidos no futuro.

De notar que este PNPAF foi destacado pela OMS como um exemplo da seguir na UE (DGS, 2016) $)^{\dagger}$.

\section{PROMOÇÃO DA SAÚDE MENTAL}

A OMS Europa acaba de lançar o "Action plan for the prevention and control of noncommunicable diseases in the WHO European Region", para promoção do consumo saudável, associado a uma política fiscal e de marketing que crie barreiras ao consumo de álcool (DGS, 2016) ${ }^{\mathrm{b}}$, um dos principais determinantes da doença mental.

Destaca-se ainda a promoção do projeto "Saúde em dia não é mania", dirigido às turmas do $12^{\circ}$ ano do Programa Integrado de Educação e Formação do Agrupamento de Escolas de Castro Verde, pela Unidade Local de Saúde do Baixo Alentejo, no seguimento das iniciativas de promoção da saúde mental na adolescência (DGS, 2016) $)^{\mathrm{m}}$ e sugere-se $\mathrm{o}$ alargamento deste projeto às outras ULS e ACES do país.

\section{PROMOÇÃO DO ENVELHECIMENTO SAUDÁVEL}

Em Portugal, de acordo com os dados estimados pelo estudo Global Burden of Diseases, os hábitos alimentares inadequados praticados por 19\% dos residentes, surgem como o fator de risco que mais contribui para o total de anos de vida saudável perdidos pela população portuguesa (DGS, 2016) ${ }^{c}$. O Programa Nacional para a Promoção da Alimentação Saudável, terá assim um papel importantíssimo no envelhecimento saudável da população. 
Em complemento, em Maio deste ano, o Governo promoveu a Estratégia Nacional para a Promoção da Atividade Física, da Saúde e do Bem-estar, que, entre outros, também será essencial para o envelhecimento saudável dos Portugueses (DGS, 2016).

Considerando que são as doenças do aparelho circulatório que mais matam em Portugal (PORDATA, 2013) e que o elevado consumo de sal está diretamente associado com estas patologias, é determinante que se implementem iniciativas que criem barreiras ao seu consumo, para que tenhamos maior esperança de vida livre de doença. De acordo com as recomendações da Organização Mundial da Saúde e da Comissão Europeia, a população portuguesa deve reduzir o consumo de sal entre $3 \%$ a $4 \%$ ao ano, durante os próximos 4 anos, procurando alcançar um consumo de 5 gramas per capita/dia até 2025. Para alcance desta meta, até ao final de 2016, o setor da restauração compromete-se a iniciar uma redução gradual e faseada do sal adicionado na confeção de alimentos (DGS, 2016) ${ }^{\circ}$.

\section{PROMOÇÃO DA UTILIZAÇÃO RACIONAL E SEGURA DO MEDICAMENTO}

De forma a promover a utilização racional de um grupo de fármacos utilizados no tratamento de situações de ansiedade e distúrbios de sono, entre outros, com elevado índice de habituação associado, os utentes integrados no Protocolo de Desabituação do Uso Crónico de Benzodiazepinas, estabelecido com o Instituto de Medicina Preventiva e Saúde Pública da faculdade de Medicina de Lisboa, foram abrangidos pelo benefício de dispensa de pagamento de taxas moderadoras, de acordo com o disposto da alínea b), do artigo 8.ำ do Decreto-Lei n.․․ 113/2011, de 29 de Novembro (ACSS, 2016) a, medida que se considera importante para o efeito pretendido.

\section{PROGRAMAS GENERALISTAS DE LITERACIA EM SAÚDE, COM IMPACTO NOS OBJETIVOS ANTERIORES}

Com o objetivo de promover a literacia em saúde, lançou-se, em Julho de 2015, o programa "Tratar de Mim". Este programa é uma iniciativa da Comissão APIFARMA OTC e tem como parceiros a Associação Nacional das Farmácias, a Direcção-Geral da Saúde, o INFARMED Autoridade Nacional do Medicamento e Produtos de Saúde, a Ordem dos Farmacêuticos, a Ordem dos Médicos e a Valormed. Este programa, que promove a literacia em Saúde junto dos mais novos, foi apoiado pelo Governo anterior e continuou a ser promovido pelo atual Governo, tendo sido apresentado, este ano, no espaço "Farmácia/Laboratório Saúde" da KidZania, em Lisboa (DGS, 2016) ${ }^{\mathrm{g}}$.

Ainda dedicado às crianças, o Programa de Alimentação Escolar, promovido pela Câmara Municipal de Lisboa, pretende implementar nas escolas de ensino público hábitos de alimentação saudável. O projeto piloto " TRINCA - uma melhor alimentação" conta com a parceria do Chef de cozinha que se desloca às escolas para preparar e confecionar ementas nutritivas de diferentes refeições (pequeno-almoço, almoço e lanche), com produtos frescos diários (DGS, 2016)i. 
Por último, informa-se que o Rovisco Pais se associou à Direção Geral de Saúde e à OMS e promoveu um evento alusivo ao Dia Mundial da Higiene das Mãos, a 5 de Maio, designado "A Saúde está nas minhas mãos" (DGS, 2016)'. Este evento considera-se importante para a prevenção de infeções, principalmente nas crianças.

\section{A OPINIÃO DE PROFISSIONAIS DE SAÚDE NO ATIVO}

O profissionais de saúde contactados indicam que as práticas de promoção da saúde e de prevenção da doença são importantíssimas para a população.

Os médicos informam-nos que, sempre que possível (desde que os familiares os acompanhem às consultas), ajudam na preparação dos prestadores informais em cuidados domiciliários quando os seus pacientes não têm capacidade ou autonomia para o fazer. Contudo, contam muitas vezes com o apoio da enfermagem para a execução deste papel, assim como para o ensino de práticas de prevenção da diabetes e da obesidade, da promoção da saúde mental e do envelhecimento saudável. Indicam que é frequente alertarem os pacientes nas consultas sobre a importância de terem um peso adequado, de fazerem exercício físico e de adotarem uma dieta o mais saudável possível e adequada ao seu estado clínico. Referem, no entanto, que é preciso maior ajuda por parte dos nutricionistas e dos dietistas neste campo, embora estejam cientes de que existem escassos recursos nas organizações públicas para contratação destes profissionais. Entendem que as Unidades de Saúde Familiares desempenham um bom papel no âmbito da promoção da saúde e da prevenção da doença, tanto ao nível da Medicina Geral e Familiar como da Enfermagem nos ACES/ULS.

Os farmacêuticos dizem ter o hábito de informar e de avaliar a utilização racional e segura do medicamento por parte dos utentes das respetivas farmácias. É ainda frequente as farmácias monitorizarem o peso, a glicémia, o colesterol, a tensão arterial, Índice de Massa Corporal e o nível de triglicéridos dos clientes com obesidade. Como grande parte dos clientes mais idosos e obesos são diabéticos, é frequente monitorizar-se, às vezes diariamente, o teor de glicose no sangue de pacientes cuja falta de sensibilidade não Ihes permite inserir as fitas nas máquinas de teste, mesmo após ensinamento repetido com a máquina do utente. Por outro lado, algumas farmácias têm consultas de podologia ou de nutrição nas suas instalações, para onde se encaminham os doentes com diabetes que possam pagar o serviço.

Embora percebam que parte dos clientes pretende a rapidez no atendimento, em detrimento da prestação de cuidados farmacêuticos no espaço farmácia, referem que cada vez mais utentes têm uma ficha de cliente onde a farmácia regista todos os dados que vai recolhendo relativamente aos pacientes, como o peso, a tensão arterial, a glicemia, o perfil lipídico, o ácido úrico, a hemoglobina, o índice de massa corporal e o perímetro abdominal. Sempre que os resultados dos testes o justifica, é solicitado aos pacientes que se dirijam ao centro de saúde ou ao hospital. Informam-nos ainda que sempre que o utente está recetivo à mensagem, o farmacêutico presta aconselhamento sobre a prevenção da doença e sobre estratégias 
Working Paper

Departamento de Ciências Económicas e Empresariais

Universidade Autónoma de Lisboa

terapêuticas sem recorrência a medicamentos de complemento à terapêutica farmacológica que acaba de aviar, tendo um papel ativo na educação para a saúde e na prestação de autocuidados.

\section{CONCLUSÃO}

Apesar dos programas prioritários definidos e implementados pelo Governo estabelecerem objetivos concretos relativamente à prevenção da diabetes e da obesidade, e à promoção da saúde mental, considera-se que é insuficiente a atenção dada ainda à preparação e apoio dos prestadores informais em cuidados domiciliários, à promoção do envelhecimento saudável e à utilização racional e segura do medicamento pela população.

Considera-se que é preciso estimular mais os profissionais de saúde de cuidados primários a educarem mais para a saúde, mas, para isso, entende-se que é necessário incentivar uma mudança de mentalidade da população. É preciso que as pessoas ponham em prática os conselhos de todas as classes de profissionais de saúde, assim como é essencial educar para que os doentes se façam acompanhar a consultas médicas quando não têm capacidade para memorizar o aconselhamento terapêutico não farmacológico.

É ainda preciso que os ACES e ULS trabalhem de forma mais próxima e articulada com as farmácias comunitárias, com as autarquias e com as escolas, no desenvolvimento de projetos de promoção da saúde. Por exemplo, vale a pena avaliar os Projectos promovidos pela Câmara Municipal de Lisboa com a Comunidade Escolar (CML, 2015) e outros, espalhados pelo território, que estejam a promover ganhos em saúde da população portuguesa.

A título de sugestão, acredita-se que o uso da aplicação MySNS, como equipamento facilitador da comunicação em promoção da saúde e em prevenção da doença para os mais jovens, seria uma mais-valia, para a implementação do PNESLA.

Por fim, não podemos esquecer a avaliação dos resultados, para a população, da implementação dos projetos descritos ao longo deste capítulo, face ao seu custo. Só assim poderemos, de futuro, fazer mais com menos, ou seja, poderemos concentrar os nossos escassos recursos nos projetos associados a mais ganhos em saúde.

\section{REFERÊNCIAS BIBLIOGRÁFICAS}

ACSS(2016)a . Protocolo de desabituação do uso crónico de benzodiazepinas $\mathrm{N}$. 8/2016/DPS/ACSS. Circular informativa. Recuperado em 5 de Outubro de 2016, de http://www.acss.min-saude.pt/Portals/0/f_512955_2.pdf

Augusto, G. e Fronteira, I. (2016). New NationalProgramme for HealthEducation, Literacyand Self-care. Online HiT. Recuperado a 5 de Outubro de 2016, de http://www.hspm.org/countries/ portugal25062012/countrypage.aspx

Câmara Municipal de Lisboa (2015). Plano de Desenvolvimento de Saúde e Qualidade de Vida da Cidade de Lisboa: Volume I- Perfil Municipal de Saúde de Lisboa. Lisboa: CML. Recuperado a 9 de Outubro de 2016, de http://lisboasolidaria.cm-lisboa.pt/documentos/ 1443532683B5fMF6 um6Ap 74HT0.pdf 
DGS (2016)a ${ }^{\text {a }}$ Programas de Saúde Prioritários. Recuperado a 5 de Outubro de 2016, de https://www.dgs.pt/programas-de-saude-prioritarios.aspx

DGS $(2016)^{\mathrm{b}}$. OMS Europa lança documento orientador para o combate às doenças crónicas. Recuperado a 5 de Outubro de 2016, de https://www.dgs.pt/em-destaque/oms-europa-lancadocumento-orientador-para-o-combate-as-doencas-cronicas.aspx

DGS (2016) c. Redução do consumo de açúcar em Portugal. Recuperado a 5 de Outubro de 2016, de https://www.dgs.pt/em-destaque/reducao-do-consumo-de-acucar-em-portugal.aspx

DGS (2016) ${ }^{d}$ Atividade física: um novo sinal vital de saúde. Recuperado em 5 de Outubro de 2016, de https://www.dgs.pt/em-destaque/atividade-fisica-um-novo-sinal-vital-de-saude.aspx

DGS (2016)e. Programas de promoção da alimentação saudável em Portugal: uma primeira proposta de avaliação. Recuperado a 5 de Outubro de 2016, de https://www.dgs.pt/emdestaque/programas-de-promocao-da-alimentacao-saudavel-em-portugal-uma-primeiraproposta-de-avaliacao.aspx

DGS (2016) Saudável. Recuperado a 5 de Outubro de 2016, de https://www.dgs.pt/em-destaque/omsdestaca-artigo-sobre-programa-nacional-para-a-promocao-da-alimentacao-saudavel-.aspx

DGS (2016)g. Jogo "Tratar de Mim" promove literacia em Saúde junto dos mais novos. Recuperado a 5 de Outubro de 2016, de https://www.dgs.pt/em-destaque/jogo-tratar-de-mimpromove-literacia-em-saude-junto-dos-mais-novos-.aspx

DGS $(2016)^{\text {h }}$. Curso online sobre alimentação saudável. Recuperado a 5 de Outubro de 2016 , de https://www.dgs.pt/em-destaque/curso-online-sobre-alimentacao-saudavel.aspx

DGS (2016)i. Chefe na escola para dar a conhecer a alimentação saudável. Recuperado a 5 de Outubro de 2016, de https://www.dgs.pt/em-destaque/chefe-na-escola-para-dar-a-conhecera-alimentacao-saudavel.aspx

DGS (2016)j. Estratégia Nacional para a Promoção da Atividade Física, da Saúde e do Bemestar. Recuperado em 5 de Outubro de 2016, de https://www.dgs.pt/emdestaque. aspx?v=41449b7a-9b2c-4d2f-9041-ce366b6bf316

DGS (2016)k. I Encontro Internacional sobre o Papel e a Importância da Família na Doença Mental. Recuperado em 5 de Outubro de 2016, de https://www.dgs.pt/em-destaque/-iencontro-internacional-sobre-o-papel-e-a-importancia-da-familia-na-doenca-mental.aspx

DGS (2016)'. A Saúde está nas minhas mãos. Recuperado a 5 de Outubro de 2016, de https://www.dgs.pt/em-destaque.aspx?v=132ae287-71a7-4c48-a3bc-963ab731a76f

DGS $(2016)^{m}$. ULSBA promove iniciativa "Saúde em Dia não é Mania". Recuperado em 5 de Outubro de 2016, de https://www.dgs.pt/em-destaque/ulsba-promove-iniciativa-saude-emdia-nao-e-mania.aspx

DGS $(2016)^{n}$. Plataforma eletrónica vai apoiar prescrição de exercício físico para doentes com diabetes. Recuperado a 5 de Outubro de 2016, de https://www.dgs.pt/emdestaque/plataforma-eletronica-vai-apoiar-prescricao-de-exercicio-fisico-para-doentes-comdiabetes-1.aspx 
DGS $(2016)^{\circ}$. Estratégias para a redução de sal nos produtos alimentares à venda em Portugal. Recuperado em 5 de Outubro de 2016, de https://www.dgs.pt/em-destaque/estrategias-paraa-reducao-de-sal-nos-produtos-alimentares-a-venda-em-portugal.aspx

DGS (2016)p. Cursos de formação para pessoas com Diabetes. Recuperado em 5 de Outubro de 2016, de https://www.dgs.pt/em-destaque/cursos-de-formacao-para-pessoas-comdiabetes. aspx

DGS (2016)a. Autoridades de Saúde Pública prosseguem visitas pedagógicas. Recuperado a 5 de Outubro de 2016, de https://www.dgs.pt/em-destaque/autoridades-de-saude-publicaprosseguem-visitas-pedagogicas.aspx

DGS (2016) r. DGS propõe redução da quantidade de açúcar nas embalagens individuais. Recuperado em 5 de Outubro de 2016, de https://www.dgs.pt/em-destaque/dgs-propoereducao-da-quantidade-de-acucar-nas-embalagens-individuais-.aspx

DGS (2015)a. Plano Nacional de Saúde 2012-2016: Revisão e Extensão a 2020. Lisboa: DGS. Recuperado em 28 de Abril de 2016, de http://pns.dgs.pt/pns-revisao-e-extensao-a-2020/.

PORDATA (2013). Óbitos por algumas causas de morte. Recuperado a 5 de Novembro de 2016, de http://www.pordata.pt/Portugal/\%C3\%93bitos+por+algumas+causas+de+morte+ (percentagem)-758

SNS (2016) a . Programa Nacional para a Saúde Mental. Recuperado em 5 de Outubro de 2016, disponível em https://www.sns.gov.pt/institucional/programas-de-saude-prioritarios/programa -nacional-para-a-saude-mental/ 